\title{
Endoscopic removal of a battery that was lodged in the oesophagus of a two-year-old boy for an extremely long time
}

\author{
Anna Szaflarska-Popławskaํ', Cezary Popławski², Bartosz Romańczuk³ ${ }^{3}$ Monika Parzęcka ${ }^{1}$ \\ 'Department of Paediatric Endoscopy and Gastrointestinal Function Testing, Nicolaus Copernicus University in Torun, \\ Ludwik Rydygier Collegium Medicum in Bydgoszcz, Poland \\ ${ }^{2}$ Department of Gastrointestinal Endoscopy, Nicolaus Copernicus University in Torun, Ludwik Rydygier Collegium Medicum \\ in Bydgoszcz, Poland \\ ${ }^{3}$ Department and Clinic of Paediatrics, Allergology, and Gastroenterology, Nicolaus Copernicus University in Torun, \\ Ludwik Rydygier Collegium Medicum in Bydgoszcz, Poland
}

Prz Gastroenterol 2015; 10 (2): 122-126 DOI: $10.5114 / p g .2014 .47497$

Key words: battery, oesophagus, endoscopy, children.

Address for correspondence: Prof. Anna Szaflarska-Popławska MD, PhD, Department of Paediatric Endoscopy and Gastrointestinal Function Testing, Nicolaus Copernicus University in Torun, Ludwik Rydygier Collegium Medicum in Bydgoszcz, 9 M. Skłodowskiej-Curie St, 85-094 Bydgoszcz, Poland, phone: +48 5258548 50, e-mail: aszaflarska@wp.pl

\begin{abstract}
In the present work we describe a 2-year-old boy whose battery ingestion was overlooked, and who had the battery endoscopically removed from the upper part of his oesophagus after several months. This is the only described case of such a long impaction of a lithium battery in the oesophagus, without development of severe complications. We stress the necessity to take into account ingestion of a dangerous foreign body by children demonstrating unspecific clinical signs.
\end{abstract}

\section{Introduction}

A consequence of the ever-growing use of small batteries in watches, hearing aids, cameras, and toys is an increasing incidence of gastrointestinal tract damage caused by battery ingestion by children, which has been observed in the last decade. In the United States, in the years 1990-2009, Hospital Emergency Departments (HEDs) recorded a total of 65,788 admissions of patients under 18 years old, due to battery ingestion (76.6\%), introduction of a battery into the oral or nasal cavity (17.7\%), or to the external auditory duct (5.7\%). During the analysed period, and in particular during the last 8 years, the number of visits due to the above-mentioned incidents has increased significantly [1]. According to a report by Centres for Disease Control and Prevention, between 1995 and 2010, 40400 children younger than 13 years old were treated in HEDs due to confirmed or anticipated incidents of battery ingestion, of which $10 \%$ required hospitalisation, and 14 children aged from 7 months to 3 years died [2].
Currently, the majority of batteries ingested by children are button batteries, which are widely used in watches, toys, cameras, calculators, and remote controllers. Depending on the applied system of electrolytes, there are different types of batteries: zinc-carbon batteries, lithium batteries, and others containing manganese dioxide, mercury oxide, silver oxide, etc. Three mechanisms of gastrointestinal tract damage resulting from battery ingestion were proposed: chemical burn due to a leak of the caustic, alkaline substance contained inside; ischaemic necrosis due to local pressure on the tissue; and production of low-voltage electric current [2-4]. Most button batteries contain a $20-45 \%$ solution of sodium or potassium hydroxide, a strong, caustic substance. The mechanism of gastrointestinal tract damage due to ingestion of such batteries is chemical burn [3]. Lithium batteries, instead of alkaline solution, contain a mildly irritating organic electrolyte, which, when leaked, does not cause topical damage. In contrast to other types of batteries, tissue damage caused by lithium batteries results mainly from the generated electric current, electrolysis of fluids, and production of hydrox- 
ides at the negative end of the battery. Therefore, the damaged tissues are the ones in contact with the negative end of the battery, and the damage may progress within days or months after removal of the battery [4].

The main factor increasing the risk of serious consequences is the long time presence of batteries (especially in the oesophagus) which is often caused by battery ingestion, which was not noticed by caretakers, and not reported by the child. Literature data indicate omission during diagnosis in at least $54 \%$ of severe and $92 \%$ of fatal complications registered after battery ingestion [4]. After an hour-long contact of the battery with the oesophagus wall, there may occur severe damage of oesophageal mucous membrane, and after $4 \mathrm{~h}$ - transmural oesophageal damage and further consequences, such as perforation, tracheobronchial fistula, damage of major blood vessels, or massive bleeding [4, 5]. A significantly more severe course can be observed in younger children, under 4 years old, and in cases of ingestion of 20-millimeter lithium batteries, especially the new type [4]. It was estimated that $12.6 \%$ of children younger than 6 years old who swallowed a 20-25-millimeter button battery will develop severe complications or die [6].

In the present work we describe a 2-year-old boy whose battery ingestion was overlooked, and who had the battery endoscopically removed from the upper part of his oesophagus after several months. This is the only described case of such a long impaction of a lithium battery in the oesophagus, without development of severe complications. We stress the necessity to take into account ingestion of a dangerous foreign body by children demonstrating unspecific clinical signs.

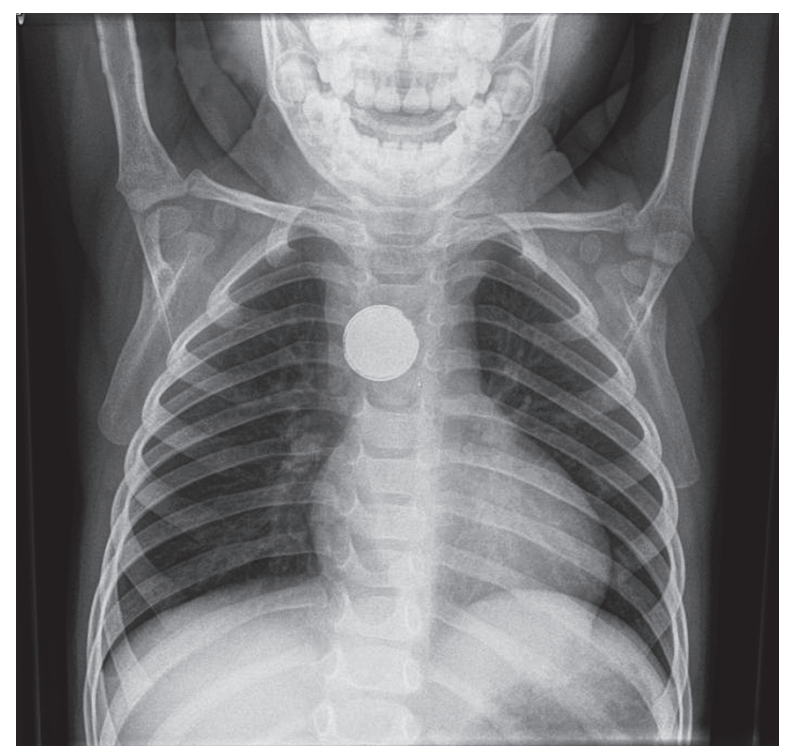

Figure 1. Foreign body in the oesophagus visible in an anterior-posterior X-ray image of the chest

\section{Case report}

A 2-year-old boy, from a unifoetal pregnancy, physiological delivery, body weight at birth 3200 g, 9 points in Apgar score, neonatal period without complications, who was hospitalised many times due to atopic dermatitis, recurring infections of the upper and lower respiratory tract, and viral infections of the gastrointestinal tract was transferred to the Department and Clinic of Paediatrics, Allergology, and Gastroenterology in Bydgoszcz from a district hospital because of a foreign body in the oesophagus. The medical history indicated that about 6 months earlier, whilst playing, the patient accidentally swallowed a button battery. Shortly after the event, the mother of the child did not observe any complaints. The first clinical signs, namely: vomiting with gastric contents a couple of times a month, mostly after substantial, solid meals; periodic uneasiness during swallowing; and poor appetite, occurred a few weeks after ingestion of the foreign body and gradually increased in subsequent months. Shortly before hospitalisation in the Clinic, the patient vomited daily, after almost every meal (solid or mush). The parents also observed difficulties with swallowing associated with strong anxiety and weeping, salivation, and refusal of solid and semi-fluid food, though with good tolerance to fluid food. During the three previous months, the boy lost $3 \mathrm{~kg}$ of weight. Moreover, during the 2 previous months the patient was treated in an outpatient clinic a few times for bronchitis and/or pneumonia diagnosed based on the clinical picture, mostly with antibiotics (X-ray image was not taken). During the stay in the district hospital, which directly preceded hospitalization in

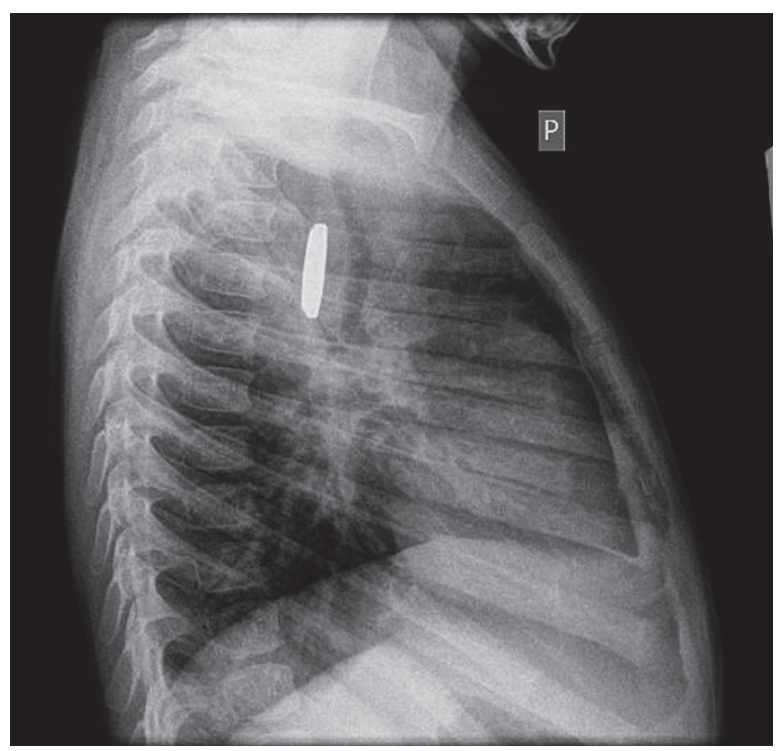

Figure 2. Foreign body in the oesophagus visible in a lateral X-ray image of the chest 


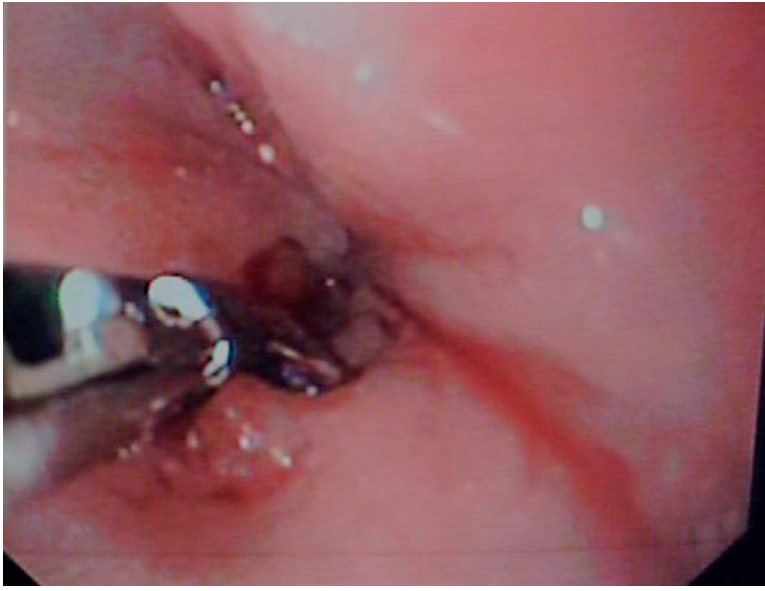

Figure 3. A nick, made by using endoscopic scissors, in the mucous membrane fold that covered the "oesophageal sack" containing the battery

the Clinic where the boy was directed due to increase in vomiting, laboratory tests showed increased ESR $(40 \mathrm{~mm} / \mathrm{h})$, and on an anterior-posterior (Figure 1) and lateral (Figure 2) chest image, a round metallic shadow of $2 \mathrm{~cm}$ in diameter, in the oesophagus, could be seen.

After admission to the Department and Clinic of Paediatrics, Allergology, and Gastroenterology in Bydgoszcz the boy's condition was assessed as fair. In physical examination, among abnormalities, anxiety, weak development of the subcutaneous tissue, and redness of the mucous membrane of the throat were found. Urgently, directly after admission of the patient, oesophagoscopy was performed under general anaesthesia, by using a GIF 160 XP endoscope (Olympus, endoscope diameter $-5.6 \mathrm{~mm}$ ), which revealed a significant stricture in the oesophagus, approximately $13 \mathrm{~cm}$ from the incisors line, with a slight showing of a metallic foreign

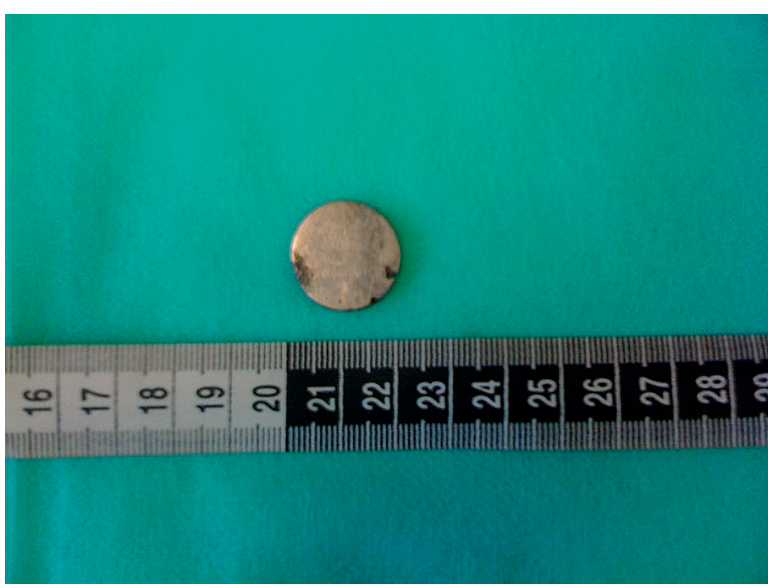

Figure 5. The lithium battery of $20 \mathrm{~mm}$ in diameter, labelled CR 2032, removed from the oesophagus

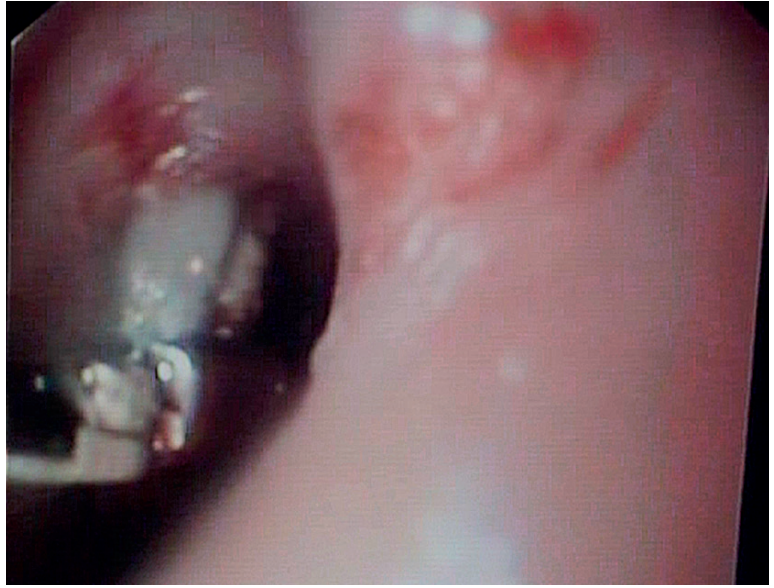

Figure 4. The battery in the "oesophageal sack" caught with alligator jaw forceps

body surrounded by a thick fold of mucous membrane. Due to the high risk of perforation, a decision was made to delay the attempt to remove the foreign body using an endoscope, until preparation of the operating room and surgical backup. Antibiotics and proton pump inhibitors were applied, and oral administration was stopped.

During the third day of hospitalisation, in an operation room setting, with surgical backup, a successful attempt was made to remove the foreign body by using a GIF 160 endoscope (Olympus). By using endoscopic scissors the thick mucous membrane fold was nicked from the upper side, which allowed access to the "sack" in the oesophagus wall, in which the battery was freely balloting. After unsuccessful attempts at catching the foreign body, or after its slipping from various endoscopic tools (Dormia basket, Roth-net, rat-tooth), the battery was ultimately caught and removed from the oesophagus by using alligator jaw forceps (Figures 3 and 4). The removal of the foreign body was complicated by a mild bleeding from the oesophageal mucous membrane. During the operation, a Flocare gastric tube of Ch 10 diameter (OvitaNutricia) was inserted using visual control. The removed button lithium battery was $20 \mathrm{~mm}$ in diameter and was labelled CR 20323 Volt (Figure 5). The X-ray images of the chest, taken directly after the operation, did not reveal any oesophageal perforation. During consecutive days of hospitalisation, intravenous therapy with antibiotics was continued, along with intragastric feeding (Nutrini, OvitaNutricia), which was well tolerated by the patient.

An endoscopic examination of the upper part of the gastrointestinal tract, which was performed 10 days later, in the location where the foreign body was lodged, in the oesophagus, adenomatous lesions were found, which narrowed and deformed the oesophageal lumen 
(Figure 6). The narrow part could easily be passed by the GIF 160 endoscope (Olympus). No abnormalities were found in the further part of the oesophagus, stomach, or duodenum. After the examination, oral feeding was included, initially with liquid and then with mush food - with a good clinical tolerance. The patient was discharged from hospital with a recommendation to perform a control visit in an outpatient gastroenterology clinic and plan the control upper gastrointestinal endoscopy.

\section{Discussion}

The present work describes a case of a 2-year-old boy for whom the diagnosis and treatment associated with ingestion of a foreign body was provided with an extremely long delay of a few months. The reason for the delay was unspecific clinical signs, which were initially mild (sporadic vomiting), but ultimately became very alarming - they made it impossible to properly feed the child, which impaired his physical development (intensive vomiting after semi-fluid and solid food, dysphagia, poor appetite, body-weight loss). There are reports in the literature that highlight unspecific clinical signs that may occur in children after battery ingestion: vomiting, abdominal pain, diarrhoea, fever, hypersomnia, irritation, and dysphagia, or signs in the respiratory system, such as cough or wheezing, and even no symptoms at all, which significantly hinders correct diagnosis and treatment. In the youngest age groups, a serious reason for delayed diagnosis is also lack of effective verbal or nonverbal communication, and sometimes fear of admitting to having swallowed a foreign body or having given it to a sibling [2]. In the described case, at the moment of confirmation of the presence of a foreign body in the oesophagus, the mother admitted that the child might have swallowed a battery approximately 6 months earlier, but it could not be determined whether she had informed general practitioners about that, and the information had been underestimated, or rather the mother had considered the swallowing of a foreign body by the child as unlikely or not harmful, and she had not sought medical assistance.

According to literature data, the presence of a battery in the oesophagus can, after just a few hours, lead to serious complications due to transmural lesions. The most serious complications reported in children include the following: tracheobronchial fistula and other perforations of the oesophagus, oesophagostenosis that requires repeated dilation, bilateral paralysis of voice chords resulting from damage of the recurrent laryngeal nerve, mediastinitis, circulatory or respiratory arrest, pneumothorax, peritonitis, tracheostenosis or tracheomalacia, aspiration pneumonia, lung abscess, or discitis.

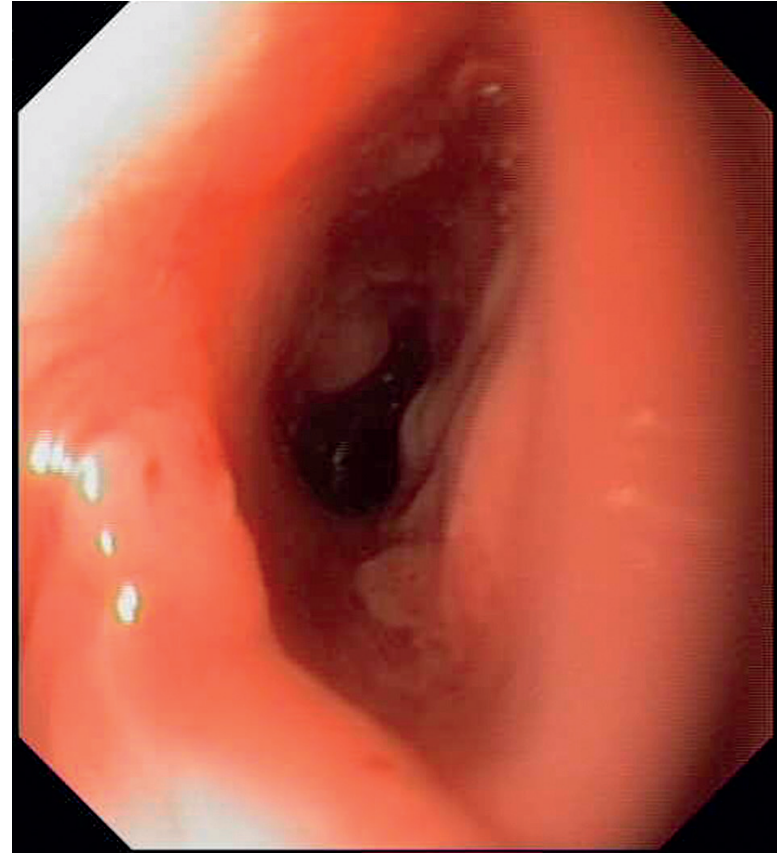

Figure 6. Stricture and deformation of the oesophageal lumen 10 days after endoscopic removal of the battery

Some of these complications can cause clinical signs after endoscopic removal of the battery: tracheobronchial fistula signs can occur within 9 days, fistulas of big blood vessels within 18 days, and oesophagostenosis within a few weeks or months $[4,6,7]$.

The two most important predictive factors of a severe course of disease are age (less than 4 years) and the size $(\geq 20 \mathrm{~mm})$ of the swallowed battery. According to Litovitz et al. [4], all fatal cases and 85-91.8\% of severe complications associated with battery ingestion involved the youngest children. In years 2000-2009, 92.1\% of batteries that were swallowed and caused severe complications or death of children were identified as 20-millimeter lithium batteries, mostly labelled as CR 2032, CR 2025, or CR2016. 12.6\% of children younger than 6 years old, who swallowed batteries of a diameter $\geq 20 \mathrm{~mm}$ died or developed severe complications. In this context, the lack of severe oesophageal and non-oesophageal complications is surprising as described in the 2-year-old boy, considering that the 20-millimeter battery was removed after a lengthy period of more than a few months. We have not found in the literature any report on a patient who had a battery in the oesophagus for such a long time. According to the data described by Litovitz et al. [4], in the group of children who died as a result of complications, the duration of the presence of a battery in the oesophagus was between $10 \mathrm{~h}$ and 2 weeks, after which time ei- 
ther the battery was removed, or the child had died. In the group of 73 children with severe complication after battery ingestion, in 72 cases the battery was removed from the oesophagus within 6 weeks, and in half of the cases - within the first day. Only in one case was the time of the battery ingestion unknown.

In the presented case, it was not determined where the battery swallowed by the child came from. According to literature data [6] 61.8\% of batteries swallowed by children younger than 6 years came directly from electronic equipment, mostly from games, toys, watches, calculators, pointers, and remote controllers; $8.2 \%$ - directly from the packaging; and $29.8 \%$ are batteries laying loose. In $70.2 \%$ of cases in which 20 -millimeter lithium batteries were ingested by children under 6 years old, if the origin of the batteries was known, they came from remote controllers. Therefore, in order to prevent children from swallowing batteries, remote controllers for TV sets, doors, garage gates, DVD players, and other devices should be considered, according to Litovitz, as requiring special supervision by caretakers of small children, and special care should be taken by the manufacturers, in order to create a protection system preventing children from easy access to batteries.

\section{Conflict of interest}

The authors declare no conflict of interest.

\section{References}

1. Sharpe SJ, Rochette LM, Smith GA. Pediatric battery-related emergency department visits in the United States, 1990-2009. Pediatrics 2012; 129: 1111-7.

2. Centers for Disease Control and prevention (CDC). Injuries from batteries among children aged $<13$ years - United States, 1995-2010. MMWR Morb Mortal Wkly Rep 2012; 61: 661-6.

3. A-Kader HH. Foreign body ingestion: children like to put objects in their mouth. World J Pediatr 2010; 6: 301-10.

4. Litovitz T, Whitaker N, Clark L, et al. Emerging battery-ingestion hazard: clinical implications. Pediatrics 2010; 125: 1168-77.

5. Kimball SJ, Park AH, Rollins MD, et al. A review of esophageal disc battery ingestions and a protocol for management. Arch Otolaryngol Head Neck Surg 2010; 136: 866-71.

6. Litovitz T, Whitaker N, Clark L. Preventing battery ingestions: an analysis of 8648 cases. Pediatrics 2010; 125: 1178-83.

7. Kim KW, Kim JY, Kim JW, et al. Compromised ventilation caused by tracheoesophageal fistula and gastrointestinal endoscope undergoing removal of disk battery on esophagus in pediatric patient - a case report. Korean J Anesthesiol 2011; 61: 257-61.

Received: 15.02.2013

Accepted: 19.03 .2013 